\title{
The PAPHIO study protocol: a randomised controlled trial with a $2 \times 2$ crossover design of physical activity adherence, psychological health and immunological outcomes in breast cancer survivors
}

\author{
Supa Pudkasam ${ }^{1,2^{*}}$ (D), Meron Pitcher ${ }^{3}$, Melanie Fisher $^{3}$, Anne O'Connor $^{4}$, Nanthaphan Chinlumprasert ${ }^{2}$,
} Lily Stojanovska ${ }^{1,5}$, Remco Polman ${ }^{6}$ and Vasso Apostolopoulos ${ }^{1 *}$

\begin{abstract}
Background: The PAPHIO study; a randomized controlled trial with 2X2 crossover design will implement a selfdirected physical activity program in which participants will engage in self-monitoring and receive motivational interviewing to enhance physical activity adherence. The study aims to determine the effects of 24 weeks selfdirected activity combined with motivational interviewing (MI) on (i) psychological health, (ii) quality of life (QoL) and (iii) immune function in female breast cancer survivors.

Methods: The study will recruit 64 female breast cancer survivors within 3 years of diagnosis and at least 6 months post primary treatments at Western Health Sunshine Hospital, Melbourne, Australia. They will be randomly allocated to immediate intervention (IIG group) or delayed intervention groups (DIG group) in a 1:1 ratio. All participants will be given a wearable device (Fitbit Alta HR) and undertake self-directed physical activity for 24 weeks and will receive MI for 12 weeks (IIG; during week 0 to week 12 and DIG; during week 13 to week 24). Participants' daily step count and the changes of immune cell functionality will be assessed at the beginning (week 1: T1), week 12 (T2) and week 24 (T3) of the program. Physical activity adherence will be assessed at T2 and T3. Participants will also complete four questionnaires assessing exercise self-regulation (BREQ2), exercise barrier and task self-efficacy, mental health (DASS-21) and QoL (FACT-B) at three time points (T1 to T3). Linear-mixed models will be used to assess the relationship between physical activity volume by step counting and mental health (DASS-21), QoL (FACTB), immune biomarkers, self-regulation (BREQ2) and self-efficacy at T1, T2 and T3;between 2 groups.
\end{abstract}

Discussion: We expect this physical activity intervention to be acceptable and beneficial to the participants in terms of psychological and immunological well-being with the potential outcomes to be implemented more widely at relatively low cost to these or other patient populations.

(Continued on next page)

\footnotetext{
* Correspondence: supa.pudkasam@live.vu.edu.au;

vasso.apostolopoulos@vu.edu.au

${ }^{1}$ Institute for Health and Sport, Victoria University, Melbourne, VIC, Australia

Full list of author information is available at the end of the article
}

(C) The Author(s). 2020 Open Access This article is licensed under a Creative Commons Attribution 4.0 International License, which permits use, sharing, adaptation, distribution and reproduction in any medium or format, as long as you give appropriate credit to the original author(s) and the source, provide a link to the Creative Commons licence, and indicate if changes were made. The images or other third party material in this article are included in the article's Creative Commons licence, unless indicated otherwise in a credit line to the material. If material is not included in the article's Creative Commons licence and your intended use is not permitted by statutory regulation or exceeds the permitted use, you will need to obtain permission directly from the copyright holder. To view a copy of this licence, visit http://creativecommons.org/licenses/by/4.0/ The Creative Commons Public Domain Dedication waiver (http://creativecommons.org/publicdomain/zero/1.0/) applies to the data made available in this article, unless otherwise stated in a credit line to the data. 
(Continued from previous page)

Trial registration: Australian New Zealand Clinical trials Registry- ACTRN12619001271190. Prospectively registered on 13 September 2019.

Keywords: Breast cancer survivor, Self-directed physical activity, Physical activity adherence, Motivational interviewing, Pedometer, Quality of life, Psychological health, And immunological biomarker

\section{Background}

Breast cancer is a common malignant disease leading to physical and psychological distress in females worldwide $[1,2]$. Approximately 2.1 million women suffered from this disease and 626,000 deceased across the world in 2018 [3]. . The occurrence rate of breast cancer has dramatically increased in 22 out of 39 countries from 2008 to 2012, whereas the global death rate has gradually dropped [4]. The global trend of breast cancer increases awareness in the availability of breast cancer care program [4].

Breast cancer survivors may have mental distress mainly due to long-term treatments [5].Currently, survivorship programs, especially physical activity programs have emphasised strategies to enhance psychosocial well-being [6, 7]. Psychological interventions including emotional ventilation, adjustment skill training and selfefficacy promotion techniques have been applied in improving their mental wellness over the decades $[6,8]$.

Physical activity, especially moderate intensity aerobic exercise for female breast cancer survivors, have been noted in a number of studies to be beneficial to breast cancer outcomes, decreasing the mortality rate by $>30 \%$ and reducing the recurrence rate [9]. As a result of physical activity, there is a reduction in the total body fat as well as a number of inflammatory and immunological biomarkers which could contribute to better outcomes in breast cancer survivors $[10,11]$. Moderate aerobic exercise and combination of aerobic and resistant training in breast cancer women ranging between 15 and 24 weeks could possibly activate immune cells such as NK cell cytotoxic activity and lymphocytes [12, 13]. Furthermore, a 12-weeks aerobic exercise training at home in breast cancer survivors reduces the level of epithelial neutrophil activating protein and pro-inflammatory cytokines [14].

Approximately one third of patients with cancer reduce their physical activity after diagnosis and almost $70 \%$ of them will not reach the exercise recommendation for cancer patients [15].. 30\% Of breast cancer survivors with early phase (stage 0 to 3 ) breast cancer have reported physical activity cessation during 12 months follow-up after participation in a 6 months RCT [16]. The cessation may be associated with the resumption of their previous domestic tasks and work [16]. Physical activity barriers in older breast cancer survivors are related to physical tiredness and lack of time management skills [17]. In addition, some breast cancer survivors have low confidence in the benefits of physical activity in minimizing adverse effects of breast cancer and treatment [18]. The challenge of recruiting patients with advanced stage of cancer is to deal with their cancer-related fatigue in particular the side effect of treatments [15].

\section{Physical activity and motivation for activity adherence in breast cancer survivors}

Adoption and adherence to physical activity programs amongst cancer survivors are challenging due to their physical and mental vulnerability [19]. As such, in this study we developed an intervention which maximizes feasibility, sustainability and generalisability. This study will prescribe self-directed physical activity to breast cancer survivors. Many of self-directed techniques for physical activity adherence in participants with advanced stage of heterogeneous cancers have been used in research studies, such as partially advised and home-based program, exercise class teaching and peer support walking group programs [20]. Many of the programs have achieved high percentages of exercise adherence, reducing fatigue and improving QoL [20].

An important factor to adopt and adhere to a physical activity program is an individual's motivation. To this end, a number of behavioural change strategies have been reported which enhance motivation and adherence [21]. Two of these strategies are Motivational Interviewing (MI) and self-monitoring. MI is a conversation technique used by a professional during consultation when making health behaviour changes [22], and has been effectively used to bring about behavioural change in health promotion programs for the general population. This includes changes in eating behaviour, alcohol cessation and adoption of an active life style [23]. More specifically, face-to-face and phone based MI has been implemented successfully to enhance selfefficacy and reduce resistance against physical activity in breast cancer survivors $[24,25]$.. On the other hand, selfmonitoring,one of the important concepts in self-regulation theory, is an auditing mechanism of individual performance in relation to an individual's cognitions, beliefs and emotions [26]. Some digital devices have the ability to promote physical activity through self-monitoring concept [27]. For example, step counting gadgets including pedometers, can effectively monitor physical activity in terms of daily steps. 
Such devices have been utilised for promotion of physical activity in clinical trials [28] especially in breast cancer patients and survivors $[29,30]$. Step counters help in monitoring individual's physical activity behaviour and can result in increased motivation and ultimately adherence to longterm physical activity behaviour [31].

To understand participants' motivation to self-directed physical activity, self-determination theory (SDT) has been effectively used to enhance insight in physical activity behaviour and motivation in breast cancer survivors [32]. According to SDT motivation to engage in behaviour lies on a continuum ranging from extrinsic (controlled by external factors) to intrinsic (individual interest and preference). The theory predicts that intrinsic-motivation might enhance confidence in task accomplishment (competence), independent action (autonomy) and the feeling of connection to others (relatedness) [32]. Our study will assess how self-regulation and motivational interviewing might change participants motivational orientation over the intervention period using the behavioural regulations in exercise questionnaire (BREQ2 [33];).

Self-efficacy, an important concept in social cognitive theory, has been shown to be a predictor for physical activity behaviour and adherence in breast cancer patient [34]. In this project two types of efficacy beliefs will be examined. First, we will explore exercise barriers self-efficacy, which will examine the participants' confidence to overcome or deal with barriers to their exercise participation. Secondly, this project will examine task self-efficacy beliefs In particular, it will examine the self-efficacy beliefs of the participants to engage in exercise behavior [34]. To measure this, the project will use the nine-item barriers self-efficacy [34] and four-item task self-efficacy questionnaire [34].

\section{Study goals and objectives}

The study has an overall aim to determine the effects of 24 weeks self-directed activity combined with motivational interviewing on (i) psychological health (depression, anxiety, stress), (ii) quality of life (QoL; physical, social/family, emotional and functional) and (iii) immune function in female breast cancer survivors. In addition, we will explore the dose-response relationship between exercise volume (step count) and the outcome measures.

\section{Study design}

This study is a randomised crossover trial; a single site research project conducted at Brest Cancer Service Clinic, Western Health (Sunshine Hospital) Australia. Potential participants will be prescribed with a Fitbit Alta HR tracker for a 24-week-self-directed activity and will receive motivational interviewing for 12 weeks (Immediate intervention group; during week 0 to week 12 and Delayed intervention group; during week 13 to week
24). The study protocol through 24 weeks is illustrated in Fig. 1.

\section{Inclusion criteria}

Female breast cancer survivors 18 years and older who are within 3 years of diagnosis and at least 6 months post active treatment; operation, chemotherapy and/or radiotherapy [stages 0 to 3 including those with ductal carcinoma in situ (DCIS)], will be recruited. Survivors on hormonal therapy such as, tamoxifen and aromatase inhibitors as well as immune targeted therapy (Herceptin) will be eligible to participate because these hormonal and cell surface blockages do not have direct effects on immune cells and adiposity-related biomarkers in breast cancer. The study will also include participants who are unable to read/ write English but are literate and English speaking. For participants with non-English literacy, translators will be used to obtain informed consent and the study will apply the questionnaires that have been validated in other languages such as Vietnamese, Greek, Chinese and Macedonian. This study plans to recruit participants from Breast cancer care service of Sunshine Hospital, Western Health, Melbourne, Australia.

\section{Exclusion criteria}

Participants who are undergoing active treatments (surgery, chemotherapy and radiation) as well as those less than 6 months post active treatment. Likewise, those individuals with cognitive impairment and unable complete the questionnaires will be excluded. Participants with known metastatic disease will also be excluded from the study. The participant will be withdrawn from the study if they develop cancer recurrence or metastases during the study period.

\section{Recruitment}

The study will recruit participants who are breast cancer survivors within 3 years of diagnosis and at least 6 months post primary treatments. Eligible participants will be randomly allocated to immediate intervention or delayed intervention groups in a 1:1 ratio after completion of informed consent. The participants will be recruited from Breast cancer care service, Western Health Sunshine campus in Melbourne. Recruitment strategies will include public media (poster advertising or flyer) and individual contact introduced by their physician [35]. All participants have to be approved by their physician for engagement in the physical activity program of this research and the screening process will be conducted before obtaining consent.

The study will commence at Western Health Sunshine campus following ethical approval from Melbourne Health Human Research Ethics Committee (MH HREC) and Western Health research governance authorization 


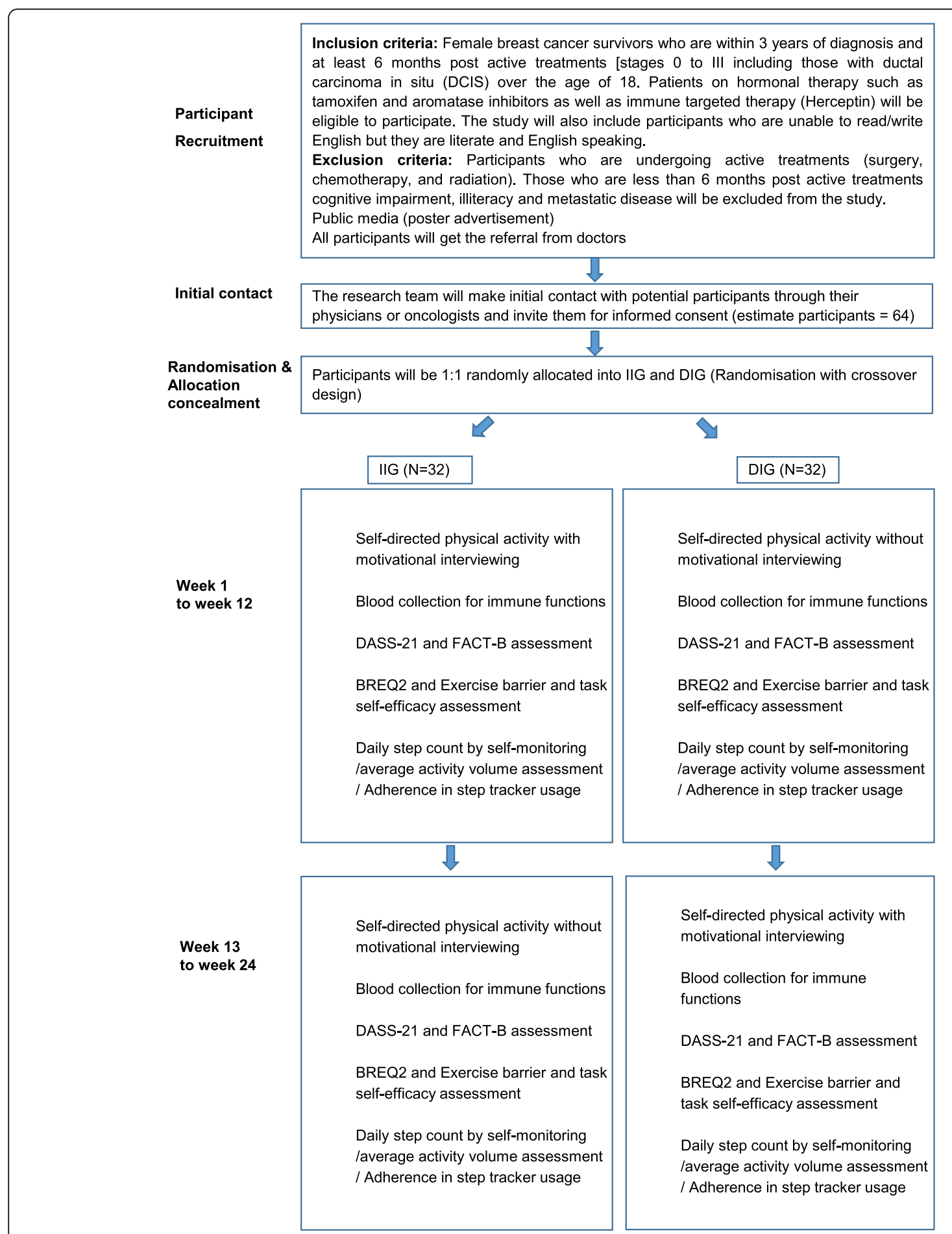

Fig. 1 Study protocol through 24 weeks trial

[36]. Based on our power calculation we aim to recruit 64 participants.

\section{Randomisation}

The study design is a two-armed RCT with a crossover design. Sequence generation will be conducted by simple randomisation following patient informed consent. The participants will be randomly allocated to either IIG or DIG in 1:1 ratio by computerised number generator. Allocation concealment by enclosing the allocation in sealed envelopes will be used to prevent selection bias [37]. The allocation concealment will be conducted by a 
third party to prevent researchers from affecting group assignment. The participants in both groups will be prescribed a self-directed physical activity for 24 weeks. The IIG will receive MI from week 1 to week 12, whereas DIG will receive the same MI intervention from weeks 13 to 24.Allocation concealment technique will be applied in process of randomisation. During weeks 1 to week 12, theIIG will perform self-directed physical activity and receive MI. On the other hand, the DIG will perform self-directed physical activity without MI. During week 13 to week 24, DIG will continue self-directed physical activity with MI. Whereas IIG will perform physical activity but will no longer be given MI.

\section{Methods of intervention}

\section{Self-directed physical activity}

Participants will perform a 24-week period of selfdirected, pedometer controlled physical activity [38, 39]. All participants will be given a step-count tracker devise (Fitbit Alta HR) and investigators will explain the prescribed pedometer-based activity to participants. Fitbit Alta HR is a wearable gadget and can be used to monitor step count. The participants' activity engagement will be accompanied by individual face-to-face and phone call MI [22]. Participants will be taught how to operate the Fitbit and will be taken on a 10 min walk to experience a moderate level of exertion.. At week 1 (baseline or T1), all participants will be prescribed to assess and record their activity using the Fitbit monitoring device through its application via computer or smartphone to establish their baseline physical activity volume by step count. Following this, they will be advised to perform their activity on their own pace as tolerated and will wear the Fitbit during the daytime or when they are available for physical activity throughout the 24 weeks. Individual participants will be advised and motivated to gradually increase their daily steps on physical activity at moderate intensity exertion or at their perception of taking some efforts but can talk during physical activity (the recommendation by the department of health, Australian government) [40] during face-to-face and over the phone MI. The participants will be advised for safety during physical activity. They will be suggested to stop physical activity if adverse symptoms occur such as chest pain or pain down to arms, dizziness, difficulty breathing, unusual rapid heart rate, and sever fatigue. The researcher will suggest to the participant to inform their family members and take a mobile phone with them before they go out to exercise. They will be also advised to take record of their daily steps in their notebook. All participants will be informed that the researcher will track the participants' step count and tracker usage time via Fitbit connect application. Individual average daily step count, and activity level will be assessed at baseline (T1), week 12 (T2), and week 24 (T3).

\section{Motivational interviewing}

MI will be used in encouraging participants through open-end questions, friendly and supportive communication and induction of behavioral changes [22, 24]. Each MI will be conducted through four phases of conversation comprising: 1) Engage, 2) Focus, 3) Evoke, and 4) Plan [22]. The study will use the dialog of MI guided by Mentha Counselling and will be conducted by a counsellor who experienced MI There will be a $20 \mathrm{~min}$ face-to-face in week 1 and three phone MI sessions (15 min) at weeks 2, 4 and 9 for IIG. For DIG this will take place at weeks 12, 13, 15 and 20.

\section{Blood collection and storage}

Approximately $20 \mathrm{ml}$ of blood will be collected via a venipuncture at T1, T2 and T3 for both groups. Whole blood will be collected into a tube containing anticoagulant and centrifuged immediately after collection or on the same day approximately $7 \mathrm{~h}$ later.

Isolation of peripheral blood mononuclear cells (PBMCs) by density gradient configuration using FicollPaque will be used for immune cell functions. PBMCs will be stored in refrigerator not longer than 1 day and then use flow cytometry to assess the composition of the isolated PBMC populations.

\section{Method of data collection and outcome measurements Primary outcomes}

Psychological health and QoL Levels of stress, depression and anxiety will be assessed using the DASS 21 [41]. The DASS21 has been validated for breast cancer survivors and has good reliability [42]. In addition, the FACT-B version 4 [43] will be used to measure QoL. This cancer specific questionnaire is well validated and has good reliability and measures physical, social/family, emotional and functional well-being aspects and breast cancer specific conditions [43, 44]. Participant will complete these instruments at baseline; week 1 (T1), week 12 (T2), and week 24 (T3).

Immune function PBMC cells (white blood cells) will be isolated from blood and PBMC assessed for changes at the cellular level by flow cytometric analysis [45] at baseline; weeks 1 (T1), 12 (T2), and 24 (T3). Cell surface markers, CD40, CD80, CD83, CD86, MHC-I, MHC-II, CD14, CD16, CD206, CD209 will be assessed by flow cytometry technique [45] to determine the changes after the program. In addition, the ratio of type 1 and type 2 $\mathrm{T}$ helper (Th1/Th2) cytokines secreted by monocytes and $\mathrm{T}$ cells will be determined to understand any cellular changes following exercise activity. 


\section{Secondary outcomes}

Average daily step count Participants will record their own daily steps through the Fitbit Alta HRapplication, upload their step count on their computer or smartphone and send the data to the researcher by email or phone call weekly. They can also record their steps from the tracker in a provided notebook. Researchers can track an individual's daily step count via Fitbit Alta HR application on a computer or smartphone. The average daily step count will be calculated at baseline at each of the 12 weeks of the intervention program.

Adherence in step tracker usage The participants' adherence to self-directed physical activity will be evaluated by their compliance by wearing the Fitbit Alta HR. The adherence rate of fitness tracker usage can be an indicator for exercise program feasibility in breast cancer patients [46]. The adherence will be defined as step count tracker wearing time with data capture (daily hours and the number of wearing days) [46]. The mean of daily hours and number of wearing days per week will be calculated at the end of week 12 and week 24 of selfdirected physical activity period [31].

Exercise self-regulation The participant's self-regulation for exercise will be assessed using the behavioral regulations in exercise questionnaire version 2 (BREQ2) [33] on three occasions. This five level-Likert scale-questionnaire has 19 items assessing exercise self-regulation which consists of five categories: external, introjected, identified, intrinsic and un-motivated [33]. The psychometric properties of the BREQ2 is adequate to assess self-regulation for exercise in breast cancer survivors [47].

Exercise barrier and task self-efficacy The nine item rating barrier self-efficacy scale will be used to assess the confidence of participants performing exercise when experienced some difficulties. Task self-efficacy will be assessed with a four items scale. This questionnaire was validated and tested for internal consistency in women with breast cancer during treatment period [34]. Efficacy beliefs will be assessed at the start and end of the trial (see study outcomes measurement in Table 1).

\section{Statistical methods \\ Sample size estimation and justification}

Based on a power of .8, alpha of .05 and large effect size in change in FACT-B (Cohen's $d=2.23$ ) from previous studies [48] we calculated that a minimum of 53 participants are required. Considering a $10-20 \%$ drop-out rate in exercise studies we decided to recruit a minimum of 64 participants ( $n=32$ in each of the two conditions).

\section{Analysis of data}

Baseline descriptive statistics (mean, standard deviation for continuous data and percentage for categorical data) will be used to describe the distribution of personal data and variables between two groups of participant (e.g., age, breast cancer health history, body compositions, and blood pressure). Independent T-test will be used to compare means for continuous data and the Pearson Chi square will be used for testing the difference in distribution of a categorical variable at baseline [49].

Linear-mixed models will be used to identify the relationship between physical activity volume by step counting and mental health (DASS-21), QoL (FACT-B), self-regulation (BREQ2) and self-efficacy at T1, T2 and T3; the relationship between activity volume by step counting and immune biomarker changes at T1,T2, and T3. The models will also be used to control for the effect of covariates or confounding factors and to manage for missing data.

\section{Data security and confidentiality}

The patient's personal information and health history which are necessary for this research study will be

Table 1 Study outcomes measurement for both groups of participant

\begin{tabular}{|c|c|c|c|c|}
\hline Outcome measurement & Method & $\begin{array}{l}\text { Week } 1 \\
\text { Baseline (T1) }\end{array}$ & $\begin{array}{l}\text { Week } 12 \\
\text { (T2) }\end{array}$ & $\begin{array}{l}\text { Week } 24 \\
\text { (T3) }\end{array}$ \\
\hline \multicolumn{5}{|l|}{ Primary outcomes } \\
\hline Psychology health & DASS-21 & $x$ & $x$ & $x$ \\
\hline QoL & FACT-B version 4 questionnaire & $x$ & $x$ & $x$ \\
\hline Immune cell functions & Isolation of peripheral blood mononuclear cells & $x$ & $x$ & $x$ \\
\hline \multicolumn{5}{|l|}{ Secondary outcomes } \\
\hline $\begin{array}{l}\text { Daily step count/Average } \\
\text { activity volume assessment }\end{array}$ & Step count tracker & $x$ & $x$ & $x$ \\
\hline Adherence in pedometer usage & $\begin{array}{l}\text { Step count tracker wearing time with data capture } \\
\text { (daily hours and the number of wearing days) }\end{array}$ & & $x$ & $x$ \\
\hline Exercise self-regulation; & BREQ2 & $x$ & $x$ & $x$ \\
\hline Exercise barrier and task self-efficacy & Exercise barrier and task self-efficacy rating scale & $x$ & $x$ & $x$ \\
\hline
\end{tabular}


protected for their privacy and confidentiality [50]. All data of participants will be recorded adequately and stored in a secure, password protected databank Agreements involving data ownership and storage will be done between Western health and Victoria University. This study has planned to hold clinical trials research data for 15 years or more based on circumstances [36]. It is possible to keep files of hard copy and electronic files in a research office at Sunshine Hospital. A locked filing cabinet and a computer for research data can only be accessed by agreed members of the research team. More specifically, the researchers will have a backup or reserved storage [36]. For destruction of the data, hard copy will be shredded by hospital and university office's shredder. Digital information will be destroyed by deleting or overwriting the files. According to the protection of participants' privacy, personal, health related data and clinical outcomes will be kept and reported in coded or reversibly anonymised technique. Re-identifiable information will be used for data management (name will be removed and replaced by a code which can be reidentified for relating the different data sets and data verification) [36].

\section{Discussion}

Self-directed physical activity in this clinical trial is considered a feasible practice in breast cancer survivors. More specifically, the combination of self-monitoring (step count) and MI have been shown to be important strategies for long-term adherence to physical activity behaviour in people living with cancer. This trial, will examine the efficacy of these health behaviour change techniques in self-directed physical activity. This is an important issue because this is a potentially low cost intervention which could be applied and implemented widely if successful. This study is guided by the SelfDetermination Theory [32] and Social Cognitive Theory [26]. We anticipate that those who adhere to the program will be more intrinsically motivated at the end of the trial in comparison to those who do not. We will also explore whether the initial motivational orientation predicts adherence to the physical activity program. In addition, we would anticipate that successful adherence to the physical activity program will be associated with higher levels self-efficacy believes and reduced number of barriers to be physically active.

The primary outcomes of this study focuses on both psychological and physiological changes which can translate positively for their breast cancer clinical outcomes. The study expects the enhancement of their QoL, psychological health and immune biomarkers in 12 to 24 weeks self-directed physical activity by pedometor application combining 12 weeks motivational interviewing.

\begin{abstract}
Abbreviations
PAPHIO study: Physical activity adherencepsychological health and immunological outcomes study; QoL: Quality of life; IIG: Immediate intervention group; DIG: Delayed intervention group; BREQ2: The behavioral regulations in exercise questionnaire version 2; DASS21: A 21-item depression, anxiety and stress scale, FACT-B: the Functional Assessment of Cancer Therapy-Breast; ANOVA: analysis of variance; MI: Motivational interviewing; PBMC: Peripheral blood mononuclear cell; ES: Effect size; HREC: Human research ethics committee; CD: Cluster of differentiation
\end{abstract}

\section{Acknowledgements}

We thank Associated Professor Michelle Ball, the Deputy Head of Psychology discipline, Victoria University for scientific peer review and Dr. Lynne Millar, senior research fellow of Australian Health Policy Collaboration, Victoria University for statistical peer review. We appreciated all comments from Melbourne Health HREC who assisted in the project protocol improvement. We also thank the office for Research at Western Health for the research governance assessment process. We appreciated Ms. Louise Bester, the manager of research contract, Victoria University for the Clinical Trial Research Agreement process. SP would like to thank Assumption University of Thailand for the postgraduate scholarship assistance. VA and SP would like to thank the Institute for Health and Sport for all their support.

\section{Authors' contributions}

VA, LS, MP, MF and AO conceived the study. VA and SP initiated research proposal, study design and protocol. RP, LS, MP, MF, AO and NC assisted and gave suggestions on the study protocol, methodology and statistical analysis. VA and SP managed the project budget. MP and MF facilitated the collaboration with breast cancer services unit at Western health. VA, MP, MF and SP amended the protocol according to Melbourne Health Human Research Ethics Committee. All authors responded to the modification of the study protocol and approved the final manuscript.

Funding

PhD research funding, Victoria University.

The funder (Victoria University taking the sponsor role of the clinical trial) has no role in the design of the study and will not have a role during project implementation, auditing, data analysis and interpretation or reporting of the study results.

\section{Availability of data and materials}

Study materials as well as datasets or analysed data during the current study are available from corresponding authors for all acceptable requests.

\section{Ethics approval and consent to participate}

The research proposal meets the requirements of the National Statement on Ethical Conduct in Human Research (2007). This study protocol (version 7: Date 15 April 2019), participant informed, consent form, recruitment and data collection materials have been approved by Melbourne Health Human Research Ethics Committee (HREC Reference Number: HREC/45268/MH-2018, Melbourne Health Site Reference Number: 2018.339) on 29 April 2019. The study is required to submit annual progress report and any proposed amendment to Melbourne Health HREC. The participants involved in this research must be well-informed and are volunteers. They all will give written consent to participate in this study.

\section{Consent for publication \\ "Not applicable".}

\section{Competing interests}

All authors declare that there are no competing interests.

\section{Author details}

${ }^{1}$ Institute for Health and Sport, Victoria University, Melbourne, VIC, Australia. ${ }^{2}$ Bernadette de Lourdes School of Nursing Science, Assumption University, Bangkok, Thailand. ${ }^{3}$ Breast Cancer Service, Western Health, Melbourne, VIC, Australia. ${ }^{4}$ IPC Health Altona Meadows, Melbourne, VIC, Australia.

${ }^{5}$ Department of Food, Nutrition and Health, College of Food and Agriculture, United Arab Emirates University, Al Ain, UAE. ${ }^{6}$ School of Exercise and Nutrition Sciences, Faculty of Health, Queensland University of Technology, Brisbane, Qld, Australia. 
Received: 30 September 2019 Accepted: 1 May 2020

\section{Published online: 15 May 2020}

\section{References}

1. Travier N, et al. Effects of an 18-week exercise programme started early during breast cancer treatment: a randomised controlled trial. BMC Med. 2015:13(1):121.

2. Sohl SJ, Levine B, Avis NE. Evaluation of the quality of life in adult Cancer survivors (QLACS) scale for early post-treatment breast cancer survivors. Qual Life Res. 2015;24(1):205-12.

3. Bray F, et al. Global cancer statistics 2018: GLOBOCAN estimates of incidence and mortality worldwide for 36 cancers in 185 countries. CA Cancer J Clin. 2018;68(6):394-424.

4. DeSantis $C E$, et al. International variation in female breast Cancer incidence and mortality rates. Cancer Epidemiol Biomark Prev. 2015:24(10):1495-506.

5. Horick NK, et al. Physical and psychological health in rare cancer survivors. J Cancer Surviv. 2017;11(1):158-65.

6. de la Torre-Luque A, et al. Psychological treatments to improve quality of life in cancer contexts: a meta-analysis. Int J Clin Health Psychol. 2016;16(2):211-9.

7. Cooney, M., et al., 'Moving on'from cancer: the effects of engaging in a 12 week community-based exercise programme on cancer survivors' physical and psychological well-being. 2016

8. Mosher $\mathrm{CE}$, et al. A systematic review of psychosocial interventions for colorectal cancer patients. Support Care Cancer. 2017;25(7):2349-62.

9. Guinan EM, Connolly EM, Hussey J. Exercise training in breast cancer survivors: a review of trials examining anthropometric and obesity-related biomarkers of breast cancer risk. Phys Ther Rev. 2013;18(2):79-89.

10. Ballard-Barbash R, et al. Physical activity, biomarkers, and disease outcomes in cancer survivors: a systematic review. J Natl Cancer Inst. 2012;104(11): 815-40.

11. Goh J, Niksirat N, Campbell KL. Exercise training and immune crosstalk in breast cancer microenvironment: exploring the paradigms of exerciseinduced immune modulation and exercise-induced myokines. Am J Trans Res. 2014;6(5):422.

12. Fairey AS, et al. Randomized controlled trial of exercise and blood immune function in postmenopausal breast cancer survivors. J Appl Physiol (1985). 2005;98(4):1534-40.

13. Hutnick NA, et al. Exercise and lymphocyte activation following chemotherapy for breast cancer. Med Sci Sports Exerc. 2005;37(11):1827-35.

14. Ergun $M$, et al. Effects of exercise on angiogenesis and apoptosis-related molecules, quality of life, fatigue and depression in breast cancer patients. Eur J Cancer Care. 2013;22(5):626-37.

15. Blaney J, et al. The cancer rehabilitation journey: barriers to and facilitators of exercise among patients with cancer-related fatigue. Phys Ther. 2016; 90(8):1135-47

16. Schmidt ME, et al. Self-reported physical activity behavior of breast cance survivors during and after adjuvant therapy: 12 months follow-up of two randomized exercise intervention trials. Acta Oncol. 2017:56(4):618-27.

17. Ventura EE, et al. Barriers to physical activity and healthy eating in young breast cancer survivors: modifiable risk factors and associations with body mass index. Breast Cancer Res Treat. 2013;142(2):423-33.

18. Hirschey R, et al. Exploration of exercise outcome expectations among breast Cancer survivors. Cancer Nurs. 2017:40(2):E39-46.

19. Jansons PS, Haines TP, O'Brien L. Interventions to achieve ongoing exercise adherence for adults with chronic health conditions who have completed a supervised exercise program: Systematic review and meta-analysis. Clin Rehabil. 2017:31(4):465-77.

20. Dittus KL, Gramling RE, Ades PA. Exercise interventions for individuals with advanced cancer: a systematic review. Prev Med. 2017:104:124-32

21. Michie $\mathrm{S}$, et al. A refined taxonomy of behaviour change techniques to help people change their physical activity and healthy eating behaviours: the CALO-RE taxonomy. Psychol Health. 2011;26(11):1479-98.

22. Miller WR, Rollnick S. Motivational interviewing. helping people change, Applications of motivational interviewing. 3rd ed. New York: Guilford Press; 2013

23. Pinto, B.M. and J.T. Ciccolo, Heldelberg: Physical activity motivation and cancer survivorship, in Physical activity and cancer. Springer. 2010. p. 36787.

24. Spencer JC, Wheeler SB. A systematic review of motivational interviewing interventions in cancer patients and survivors. Patient Educ Couns. 2016; 99(7):1099-105.
25. Sheppard VB, et al. The feasibility and acceptability of a diet and exercise trial in overweight and obese black breast cancer survivors: the stepping STONE study. Contemporary Clin Trials. 2016;46:106-13.

26. Bandura A. Social cognitive theory of self-regulation. Organ Behav Hum Decis Process. 1991;50(2):248-87.

27. Lewis BA, et al. Future directions in physical activity intervention research: expanding our focus to sedentary behaviors, technology, and dissemination. J Behav Med. 2017;40(1):112-26.

28. Tudor-Locke C, et al. How many steps/day are enough? For older adults and special populations. Int J Behav Nutr Phys Act. 2011;8:80.

29. Brunet J, Burke SM, Sabiston CM. The benefits of being self-determined in promoting physical activity and affective well-being among women recently treated for breast cancer. Psychooncology. 2013;22(10):2245-52

30. Rogers LQ, et al. Effects of the BEAT Cancer physical activity behavior change intervention on physical activity, aerobic fitness, and quality of life in breast cancer survivors: a multicenter randomized controlled trial. Breast Cancer Res Treat. 2015;149(1):109-19.

31. Cadmus-Bertram $L$, et al. Use of the Fitbit to measure adherence to a physical activity intervention among overweight or obese, postmenopausal women: self-monitoring trajectory during 16 weeks. JMIR Mhealth And Uhealth. 2015;3(4):e96.

32. Milne HM, et al. Self-determination theory and physical activity among breast cancer survivors. J Sport Exerc Psychol. 2008;30(1):23-38.

33. Markland D, Tobin V. A modification to the behavioural regulation in exercise questionnaire to include an assessment of amotivation. J Sport Exerc Psychol. 2004;26(2):191-6.

34. Rogers $L Q$, et al. Exercise barrier and task self-efficacy in breast cancer patients during treatment. Support Care Cancer. 2006;14(1):84-90.

35. Deakin University, Recruiting human participants. 2016.

36. National Health and Medical Research Council. National statement on ethical conduct in human research 2007. 2015.

37. Consort group. Consort transparent reporting of trials. 2010.

38. Takacs J, et al. Validation of the Fitbit one activity monitor device during treadmill walking. J Sci Med Sport. 2014;17(5):496-500.

39. Hartman SJ, et al. Impact of increasing physical activity on cognitive functioning in breast cancer survivors: rationale and study design of memory \& motion. Contemporary Clin Trials. 2015:45:371-6.

40. Department of health Australian Government. Choose health: be active: a physical activity guide for older Australians. 2009.

41. Lovibond PF, Lovibond SH. The structure of negative emotional states: comparison of the depression anxiety stress scales (DASS) with the Beck depression and anxiety inventories. Behav Res Ther. 1995;33(3):335-43.

42. Schreiber JA. Image of God: effect on coping and psychospiritual outcomes in early breast cancer survivors. in Oncology Nursing Forum; 2011.

43. Garcia SF, Hahn EA. Measure validation is an ongoing process: the functional assessment of Cancer therapy-breast symptom index as a case example. Ann Palliat Med. 2012;1(3):207-10.

44. Brady MJ, et al. Reliability and validity of the functional assessment of Cancer therapy-breast quality-of-life instrument. J Clin Oncol. 1997;15(3): 974-86.

45. Han $\mathrm{TH}$, et al. Evaluation of 3 clinical dendritic cell maturation protocols containing lipopolysaccharide and interferon-gamma. J Immunother (Hagerstown 1997). 2009;32(4):399-407.

46. De Jesus $\mathrm{S}$, et al. Feasibility of an exercise intervention for fatigued breast cancer patients at a community-based cardiac rehabilitation program. Cancer Manag Res. 2017;9:29-39.

47. Milne HM, et al. Impact of a combined resistance and aerobic exercise program on motivational variables in breast Cancer survivors: a randomized controlled trial. Ann Behav Med. 2008:36(2):158-66.

48. Milne HM, et al. Effects of a combined aerobic and resistance exercise program in breast cancer survivors: a randomized controlled trial. Breast Cancer Res Treat. 2008;108(2):279-88.

49. Hazra A, Gogtay N. Biostatistics series module 4: comparing groups categorical variables. Indian J Dermatol. 2016;61(4):385-92.

50. World Health Association, World Medical Association Declaration of Helsinki Ethical Principles for Medical Research Involving Human Subjects. 2016.

\section{Publisher's Note}

Springer Nature remains neutral with regard to jurisdictional claims in published maps and institutional affiliations. 